\title{
Is a single direct MR arthrography series in ABER position as accurate in detecting anteroinferior labroligamentous lesions as conventional MR arthography?
}

\author{
Saskia A. Schreinemachers • \\ Victor P. M. van der Hulst • W. Jaap Willems • \\ Shandra Bipat $\cdot$ Henk-Jan van der Woude
}

Received: 31 December 2008 /Revised: 4 March 2009 / Accepted: 22 March 2009/Published online: 7 May 2009

(C) The Author(s) 2009. This article is published with open access at Springerlink.com

\begin{abstract}
Purpose The purpose of this study is to retrospectively compare accuracy of single magnetic resonance (MR) arthrography series in Abduction External Rotation (ABER) with conventional MR arthrography for detection and characterisation of anteroinferior labroligamentous lesions, with arthroscopy as reference standard. Interobserver variability of both protocols was determined.

Methods and materials Institutional review board approval was obtained; informed consent was waived. MR arthrograms, including oblique axial fat suppressed T1-weighted images in ABER position and conventional imaging directions of 250 patients (170 men, 80 women; mean age, 36 years), were retrospectively and independently evaluated by three reviewers. Reviewers were blinded to clinical information and arthroscopic results. Labroligamentous lesions were registered in both ABER and MRa. The lesions were sub-classified (Bankart, Perthes, anterior labrum periosteal sleeve avulsion (ALPSA) or lesions not otherwise specified). Inter-observer agreement was assessed
\end{abstract}

\footnotetext{
S. A. Schreinemachers $(\bowtie) \cdot$ V. P. M. van der Hulst •

H.-J. van der Woude

Department of Radiology, Onze Lieve Vrouwe Gasthuis,

Oosterpark 9, Postbus 95500,

1090 HM Amsterdam, The Netherlands

e-mail: sschreinemachers@gmail.com

W. Jaap Willems

Orthopaedic Surgery, Onze Lieve Vrouwe Gasthuis,

Oosterpark 9, Postbus 95500,

1090 HM Amsterdam, The Netherlands

S. Bipat

Department of Radiology, Academic Medical Centre,

University of Amsterdam,

Amsterdam, The Netherlands
}

by Kappa statistics for all 250 patients. Ninety-two of 250 patients underwent arthroscopy. Sensitivity, specificity and accuracy of ABER versus conventional MR arthrography were calculated and compared using paired McNemar test. Results Kappa values of the ABER and conventional MR arthrography ranged from 0.44 to 0.56 and 0.44 to 0.62 , respectively. According to arthroscopy, 45 of 92 patients had an intact anteroinferior labrum, and in 44 patients, a labroligamentous lesion (eight Bankart, seven Perthes, 29 ALPSA and three lesions not otherwise specified) was diagnosed. There were no statistically significant differences between ABER and conventional MR arthrography regarding sensitivity $(85-89 \%, 89-96 \%)$, specificity $(82-$ $91 \%, 84-89 \%)$ and overall accuracy $(50-62 \%, 53-63 \%)$.

Conclusion The results of a single MR arthrography series in ABER position are comparable with those of conventional MR arthrography for detecting anteroinferior labroligamentous lesions.

Keywords Shoulder joint - Shoulder instability . Magnetic resonance imaging · Arthrography $\cdot$ ABER

\section{Introduction}

Glenohumeral instability is a common disorder of the shoulder and results from an imbalance between the static and dynamic stabilisers in and around the glenohumeral joint [1].

Glenohumeral instability can be categorised as atraumatic (due to laxity of ligaments), microtraumatic (secondary to repetitive overhead movements or overuse) and traumatic [2]. The instability due to trauma is usually in anterior direction and may lead to a variety of anteroinferior 
labroligamentous lesions. Bankart, Perthes and Anterior Labrum Periosteal Sleeve Avulsion (ALPSA) lesions are by far the most frequently encountered lesions in patients with anterior shoulder instability, whereas humeral avulsion glenohumeral ligament (HAGL) and glenolabral articular disruption lesions (GLAD) are much less common. Surgery is often required after multiple dislocations. Successful open or arthroscopic treatment requires a thorough preoperative evaluation of the anatomic structures being involved.

Direct magnetic resonance (MR) arthrography is considered to be the most accurate imaging technique for visualisation of the anteroinferior labroligamentous complex because of optimal joint distention with a diluted mixture of Gd-DTPA and superb soft tissue contrast [3-10].

During the last decade, different areas for improvement of shoulder MR arthrography have been investigated. In an attempt to increase the detection rate of labroligamentous tears, the usefulness of different variations in arm positioning have been explored, such as adduction internal rotation [11] and the apprehension test position with $90^{\circ}$ abduction and maximal tolerable external rotation [12]. Imaging with the arm positioned in abduction and exorotation (ABER) has been more comprehensively explored. Different studies described an increase in accuracy of MR arthrography when a sequence obtained in ABER position was added to a series of conventional sequences [12-16].

The use of additional series to a routine MR arthrography protocol in neutral shoulder position is not widespread. More time is required for repositioning the shoulder coil and performing an additional survey scan. Moreover, patients may not tolerate ABER positioning because this may provoke glenohumeral dislocation.

To improve diagnostic accuracy of MR arthrography with shortening of scan time is challenging. To our knowledge, only two (in vivo) studies [14, 16] reported on the comparison of conventional MR arthrography with a single fat-suppressed T1-weighted series in ABER position.

The purpose of this study was to retrospectively compare accuracy of a single MR arthrography series in ABER with conventional MR arthrography for the detection and characterization of anteroinferior labroligamentous lesions with arthroscopy as reference standard. Inter-observer variability of both protocols was determined.

\section{Material and methods}

\section{Patients}

This retrospective study was approved by the local medical ethics committee, and patient informed consent was waived. Two hundred fifty consecutive patients who underwent MR arthrography of the shoulder at our institution between January 2001 and July 2003 were included. Patients were included when a complete examination was available including T1- and T2-weighted sequences in axial, oblique coronal and oblique sagittal directions and an oblique axial fat-suppressed T1-weighted sequence in ABER position.

The medical records were reviewed to identify the reason for referral including complaints of shoulder impingement, instability or pain of unknown origin. Of the 250 patients, 134 had been referred for instability, 64 patients for clinical signs of impingement and 52 patients for pain of unknown origin. Fourteen patients had undergone surgery to the symptomatic shoulder previously.

Imaging technique

All patients underwent intra-articular administration of $10 \mathrm{~mL}$ omnipaque 300 (300 mg I/ml iohexol; GE Healthcare $\mathrm{BV}$, Eindhoven) and a $10-\mathrm{mL}$ mixture of $0.5 \mathrm{~mL}$ omniscan (0.5 mmol/ml Gd-DTPA-BMA; GE Healthcare $\mathrm{BV}$, Eindhoven) added to $100 \mathrm{~mL}$ saline $0.9 \%$. Of this solution, $12-15 \mathrm{~cm}^{3}$ was administered after inserting an 18-gauge needle in the glenohumeral joint with fluoroscopic guidance, using either an anterior or a posterior approach. MR imaging was obtained within 30 min (after injection, patients were instructed to immobilise the shoulder of interest until the MR examination).

Imaging was performed with either a 1.0-T unit (MR Systems NT Release 4.5; Philips Medical Systems, Best, The Netherlands) or 1.5-T unit (MR Systems Intera, Release 9.0, Philips Medical Systems, Best, The Netherlands).

The following imaging protocol in neutral position (conventional MR arthrography) was used: T1-weighted fast field echo (381.5-400/9.21-13.8 millisecond repetition time (RT)/echo time (TE), 4-mm section thickness, 0.4 gap, NSA 3 ) in the true axial plane. Proton density turbo spin echo (TSE) (2000/15-30 ms TR/TE, 3- to 4-mm section thickness, 0.3 to 0.4 gap, NSA 2), T2-weighted TSE (3021.8-3058/90 ms TR/TE, 3- to 4-mm section thickness, 0.3 to 0.4 gap, NSA $3(1.0 \mathrm{~T})$ or $4(1.5 \mathrm{~T}))$ and T1-weighted fat-suppressed TSE (500/16 TR/TE, 4-mm section thickness, 0.4 gap, NSA $2(1.0 \mathrm{~T})$ or $3(1.5 \mathrm{~T}))$ in the oblique coronal plane. T1-weighted fat-suppressed TSE (500/14$16 \mathrm{~ms} \mathrm{TR} / \mathrm{TE}, 3$ - to $4-\mathrm{mm}$ section thickness, 0.3 to 0.4 gap, NSA $2(1.0 \mathrm{~T})$ or $3(1.5 \mathrm{~T}))$ in the oblique sagittal plane. All sequences were acquired with a $256 \times 256$-matrix and 120 / $160 \times 75 / 100-\mathrm{mm}$ field of view. A C3/C1 surface coil $(1.0 \mathrm{~T})$ or a Synergy Flex-M coil $(1.5 \mathrm{~T})$ was positioned on the shoulder. Total scan time was $18: 46 \mathrm{~min}(1.5 \mathrm{~T})$ and 18:08 $\min (1.0 \mathrm{~T})$, respectively, patient and coil positioning not included.

To obtain the ABER position [17], the ipsilateral hand was positioned posteriorly to the head with the elbow 
positioned towards the table. The surface coil was repositioned accordingly.

T1-weighted fat-suppressed TSE (500-525/14-17 TR/ TE, 3-mm section thickness, 0.3 gap, 120/160 $\times 100$ FOV, $256 \times 256$ matrix and two $(1.0 \mathrm{~T})$ to three $(1.5 \mathrm{~T})$ signals acquired) in an oblique axial plane. Additional scan time was 5:07 $\mathrm{min}(1.5 \mathrm{~T})$ and 4:41 $\min (1.0 \mathrm{~T})$, respectively, patient and coil repositioning not included.

\section{Image analysis}

For image analysis, all ABER series were separated from the images in neutral position and were placed in random order. This was done by one investigator (S.A.S.) who was not involved in the image interpretation.

Two musculoskeletal radiologists (reviewer 1: H.J.W. and reviewer 2: V.P.M.H.) with at least 12 years of experience on MRI of the musculoskeletal system and one orthopaedic surgeon (reviewer 3: W.J.W.) with 12 years experience on shoulder MR arthrography independently reviewed the MR arthrography studies in random order. The reviewers were blinded to the patient's personal details, clinical history and symptoms and, if any, the arthroscopic results.

Anteroinferior labroligamentous lesions were registered as absent or present. In case of presence of anteroinferior labroligamentous lesions, this was classified as a Bankart, Perthes, ALPSA, as these lesions are by far most commonly encountered in patients with shoulder instability, or lesions not otherwise specified (NOS).

The definitions of the lesions were as follows:

Bankart an avulsion of the anteroinferior labrum from the glenoid with a disrupted periosteum [18]

Perthes an avulsion of the anteroinferior labrum from the glenoid with an intact periosteum, which is stripped medially [19]

ALPSA an avulsion of the inferior glenohumeral labroligamentous complex with medially displacement and inferiorly rotation of the avulsed labrum to the glenoid with an intact periosteum [20]

Examples of MR arthrography images in neutral and ABER position for each type of anteroinferior labroligamentous lesions are demonstrated in Figs. 1, 2, and 3.

\section{Reference standard}

To determine the accuracy of MR arthrography for both conventional MR arthrography and ABER series, the patients who underwent an arthroscopy within a maximum time interval of 12 months between MR and arthroscopy were identified. Of 250 patients, 92 underwent arthroscopy
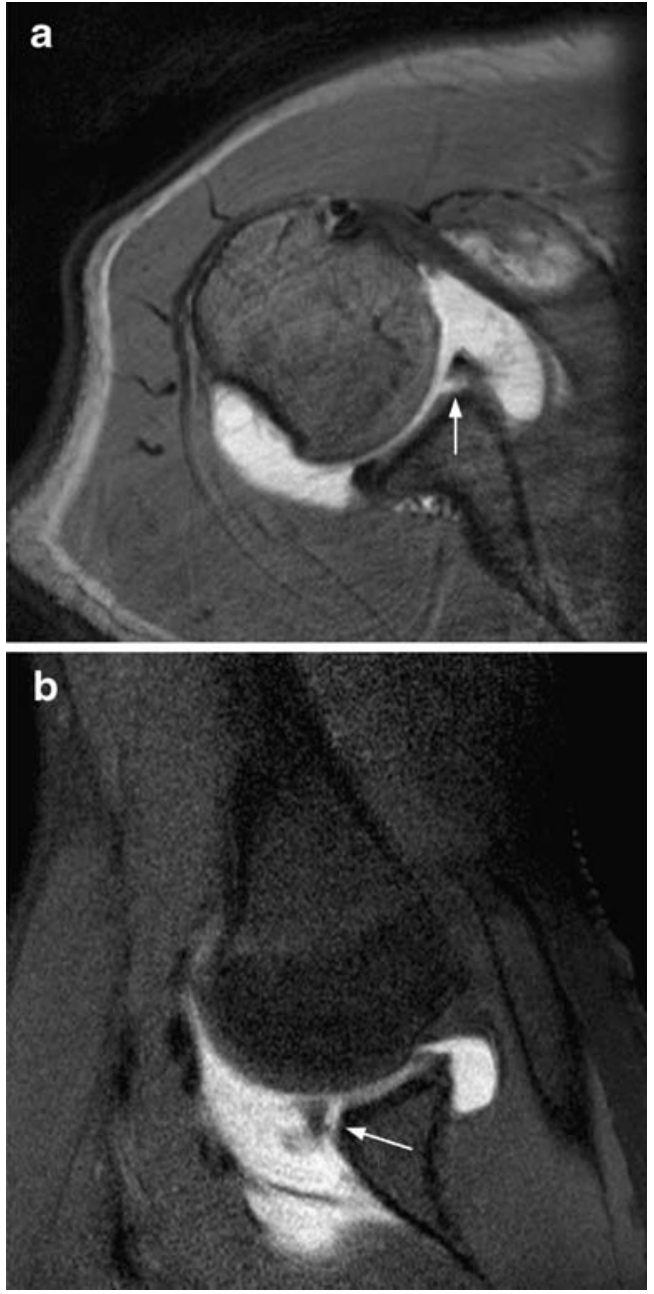

Fig. 1 Bankart lesion. a T1-weighted fast field echo (FFE) MR arthrography image in an axial plane at the level of the inferior glenoid labrum with the arm in neutral position; avulsion of the anteroinferior labrum with stripping and complete disruption of the periosteum (arrow). b T1-weighted fat suppressed turbo spin echo (TSE) MR arthrography image in an oblique axial plane at the level of the inferior glenoid labrum with the arm positioned in abduction and exorotation (ABER) demonstrates the avulsed anteroinferior labrum with stripping and complete disruption of the periosteum (arrow). Note the bony glenoid defect

within this interval (Fig. 4). The surgical records of these 92 patients were reviewed by one investigator (S.A.S.) - who was not involved in the image interpretation for this studyto identify the arthroscopic results.

Shoulder arthroscopies were performed in lateral decubitus position under general anaesthesia. Routinely two portals were used to enable a thorough arthroscopic evaluation of the glenohumeral joint and subacromial space. In case of surgery (instability repair, subacromial decompression or cuff repair) additional portals were used.

At arthroscopy anteroinferior labroligamentous lesions were classified as Bankart, ALPSA, Perthes or lesions not otherwise specified. 

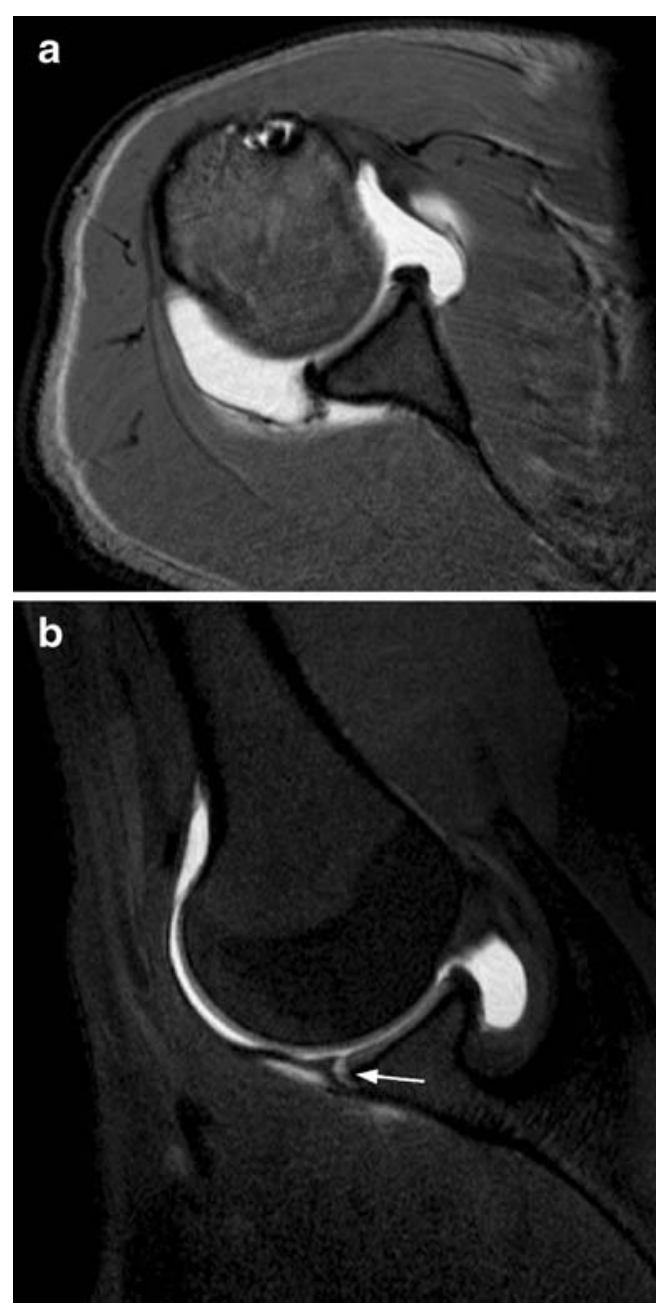

Fig. 2 Perthes lesion. a T1-weighted FFE MR arthrography image in an axial plane at the level of the inferior glenoid labrum with the arm in neutral position demonstrates no obvious anteroinferior labroligamentous lesion. b T1-weighted fat suppressed TSE MR arthrography image of the same patient in an oblique axial plane in ABER position clearly demonstrates the avulsed anteroinferior labrum with medial stripping of an intact periosteum (arrow)

\section{Statistical analysis}

\section{Inter-observer variability}

The inter-observer agreement was assessed by Kappa statistics, and agreements in percentages were calculated for all 250 patients.

\section{Diagnostic accuracy}

Imaging findings at MR arthrography (ABER and conventional MR arthrography) and arthroscopic results were compared in 92 of 250 patients for calculation of sensitivity, specificity and accuracy. Agreement between MR arthrographic and arthroscopic registration of the presence or absence of anterior labroligamentous lesions was obtained with a sensitivity and specificity. Accuracy was defined as correct classification, i.e., normal labrum, Bankart, Perthes and ALPSA lesion. Sensitivity, specificity and accuracy values of the ABER and conventional MR arthrography were compared using the paired Mc Nemar test; a $p$ value $<0.05$ was considered a statistically significant difference.

All analyses were performed with SPSS 12.0.1 software (SPSS, Chicago, IL).

\section{Results}

\section{Patients}

Two hundred fifty MR arthrograms of 250 patients were reviewed. In 92 of 250 patients, an arthroscopy was
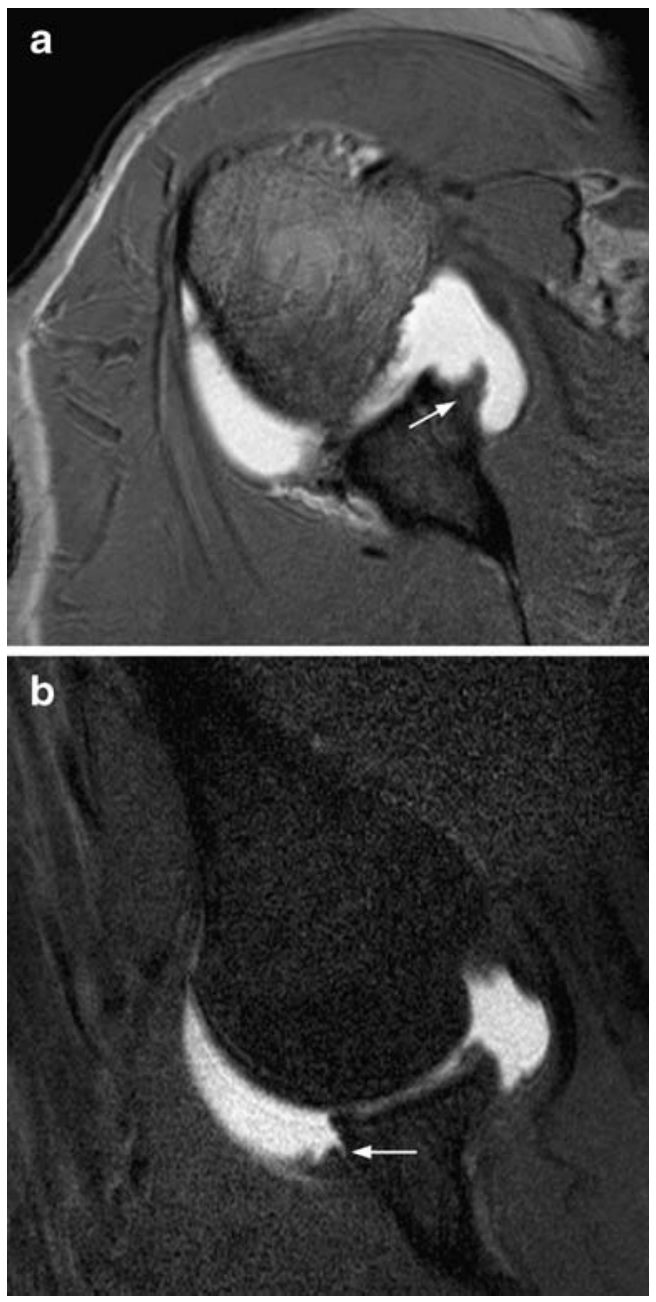

Fig. 3 Anterior Labrum Periosteal Sleeve Avulsion. a T1-weighted FFE MR arthrography image in an axial plane at the level of the inferior glenoid labrum with the arm in neutral position demonstrates a medially displaced and inferiorly rotated labrum (arrow). b T1weighted fat suppressed TSE MR arthrography image in an oblique axial plane with the arm in ABER position shows a medially displaced and inferiorly rotated anteroinferior labrum (arrow) 


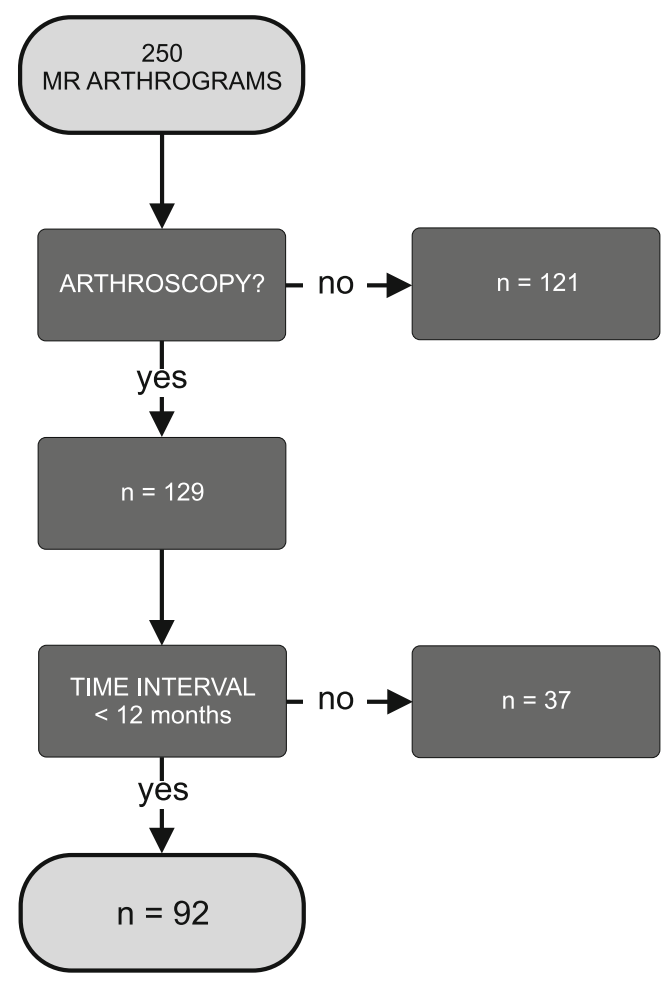

Fig. 4 Patient selection and inclusion

performed within a maximum time interval of 12 months. The baseline patient characteristics are listed in Table 1. No differences in patient characteristics could be observed between the total group and the patients who underwent arthroscopy.

MR Arthrography findings (250 MR arthrograms)

One hundred thirty (52\%) of $250 \mathrm{MR}$ arthrograms were performed on the $1.0 \mathrm{~T}$ unit and $120(48 \%)$ on the $1.5 \mathrm{~T}$ unit.

The MR arthrography results as scored by each individual observer for the total group of patients $(n=250)$ are shown in Table 2. Bankart lesions and ALPSA lesions were predominantly found using the conventional MR

Table 1 Baseline patient characteristics

\begin{tabular}{|c|c|c|}
\hline & $\begin{array}{l}250 \mathrm{MR} \\
\text { arthrograms }\end{array}$ & $\begin{array}{l}\text { Study group (92 } \\
\text { MR arthrograms) }\end{array}$ \\
\hline Male/Female & $170 / 80$ & $61 / 31$ \\
\hline Age in years: mean \pm SD (range) & $35.6(12.8)$ & $34.0(12.5)$ \\
\hline \multicolumn{3}{|l|}{ Clinical signs/symptoms } \\
\hline Impingement & $64(25.5 \%)$ & $28(30 \%)$ \\
\hline Instability & $134(53.5 \%)$ & $58(63 \%)$ \\
\hline Pain of unknown origin & $52(21 \%)$ & $6(7 \%)$ \\
\hline $\begin{array}{l}\text { Previously surgery to the } \\
\text { symptomatic shoulder }\end{array}$ & $14(6 \%)$ & $8(9 \%)$ \\
\hline
\end{tabular}

arthrography. Varying from 29 to 75 Bankart lesions were diagnosed on the MRa versus 19 to 49 on the ABER series. Varying from 33 to 75 ALPSA lesions were found on the conventional MR arthrography versus eight to 44 on the ABER series. Perthes lesions were particularly noticed in the ABER group: 21 to 45 Perthes lesions on the ABER series versus two to five perthes lesions on the conventional MR arthrography.

According to one or two reviewers in nine out of 250 patients the ABER and/or conventional MR arthrography was not of sufficient quality to evaluate the anteroinferior labrum. Reviewer 1 classified one anteroinferior labrum on both ABER and conventional MR arthrography as 'not assessable' and according to reviewer 3 seven ABER and two conventional MR arthrography series were not assessable, all because of insufficient amount of intra-articular contrast medium, motion artefacts and/or incorrect positioning of the surface coil.

\section{Inter-observer variability}

The results of the inter-observer variability with regard to the detection and characterization of anterior labrum lesions are shown in Table 3. Kappa values of ABER and conventional MR arthrography ranged from 0.44 to 0.56 and from 0.44 to 0.62 , respectively. Agreements ranged from 67 to 75 and from 66 to 78 , respectively.

MR Arthrography versus Arthroscopy (study group $n=92$ )

In 92 of 250 patients an arthroscopy was performed within 12 months between MR arthrography and arthroscopy. The number of patients performed on the 1.0 and $1.5 \mathrm{~T}$ units was equal in this study group.

According to the reference standard, 45 patients had an intact anteroinferior labrum, eight had a Bankart lesion, seven patients had a Perthes lesion and in 29 patients, an ALPSA lesion was determined. In three patients, the labral lesion was not otherwise specified because of either a hardly arthroscopically recognisable anteroinferior labrum (twice) or an eroded anteroinferior labrum in three shoulder joints showing a detached joint capsule.

The results of the MR arthrography in ABER position versus neutral position are shown in Tables 4 and 5 , respectively. According to reviewer 1, 47 out of 92 patients had an intact anteroinferior labrum on ABER versus 45 out of 92 on conventional MR arthrography and in 44 out of 92 an anteroinferior labroligamentous lesion was classified on ABER versus 46 on conventional MR arthrography. According to reviewer 1 , four out of 44 lesions were unclassifiable using the ABER and one out of 46 using the conventional MR arthrography. According to reviewers 2 and 3, respectively, 42 out of 92 and 46 out of 92 had an 
Table 2250 MR arthrograms: results of ABER and conventional MR arthrography obtained by each reviewer

\begin{tabular}{|c|c|c|c|c|c|c|}
\hline & Intact & Bankart & Perthes & ALPSA & Lesions NOS & Not assessable \\
\hline ABER & $159 / 148 / 154$ & $23 / 49 / 19$ & $21 / 45 / 26$ & $40 / 8 / 44$ & $6 / 0 / 0$ & $1 / 0 / 7$ \\
\hline conventional MR arthrography & $149 / 139 / 138$ & $44 / 75 / 29$ & $4 / 2 / 5$ & $48 / 33 / 75$ & $4 / 1 / 1$ & $1 / 0 / 2$ \\
\hline
\end{tabular}

The results of reviewer 1 are listed before the first slash, of reviewer 2 between the two slashes and the results of reviewer 3 behind the second slash.

intact anteroinferior labrum on ABER versus 40 out of 92 and 41 out of 92 on conventional MR arthrography. In 50 out of 92 and 43 out of 92 patients, a labrum lesion was diagnosed on ABER versus 52 out of 92 and 41 out of 92 on conventional MR arthrography. According to reviewer 3 , one out of 43 lesions diagnosed on conventional MR arthrography was unclassifiable.

In four out of 92 patients, one or two reviewers classified the ABER or conventional MR arthrography series as 'not assessable', because of insufficient amount of intra-articular contrast medium, motion artefacts and/or incorrect positioning of the surface coil. Reviewer 1 classified one labrum on both ABER and conventional MR arthrography as 'not evaluable' and reviewer 3 classified three MR arthrograms in ABER position and one conventional MR arthrography as 'not assessable'.

\section{Sensitivity/specificity}

Sensitivity and specificity numbers regarding the assessment of presence or absence of anteroinferior labroligamentous lesions using MR arthrography compared with arthroscopy are summarised in Table 6. Lesions not assessable on MR arthrography, if any (see Tables 4 and 5), were regarded as false negative results in the calculation of sensitivity values and as false positive results in the calculation of specificity values.

According to arthroscopy 45 of 92 patients had an intact anteroinferior labrum and in 47 patients, an anteroinferior labrum lesion was diagnosed.

The calculated sensitivity and specificity values of ABER were 40/47 and 40/45 (reviewer 1), 42/47 and 37/ 45 (reviewer 2) and 41/47 and 41/45 (reviewer 3). The calculated sensitivity and specificity values of conventional
MR arthrography were $42 / 47$ and 40/45 (reviewer 1), 45/47 and 38/45 (reviewer 2) and 43/47 and 38/45 (reviewer 3), respectively (Table 6). In nine patients, ABER assessments proved to be false negative. These scores were done in the same particular patients by at least two reviewers in six out of nine.

The $p$ values based on the sensitivity and specificity values of ABER and conventional MR arthrography were 0.69 and 1.0 (reviewer 1), 0.38 and 1.0 (reviewer 2) and 0.75 and 0.45 , respectively (reviewer 3 ). All $p$ values exceed 0.05 ; therefore, no significant difference was found between the sensitivity and specificity of the ABER and conventional MR arthrography.

\section{Accuracy}

Based on the single series in ABER position, the correct classification of normal anteroinferior labrum, Bankart, Perthes and ALPSA lesion for reviewer 1 was 57/92 (62\%), for reviewer $246 / 92(50 \%)$ and for reviewer $356 /$ 92 (61\%; see Table 4). Based on the conventional MR arthrography, the correct classification of normal anteroinferior labrum, Bankart, Perthes and ALPSA lesion for reviewer 1 was 58/92 (63\%), for reviewer 2 49/92 (53\%) and for reviewer $362 / 92$ (67\%; see Table 5).

The $p$ values based on the accuracy values of both examinations were 0.58 (reviewer 1), 0.63 (reviewer 2) and 0.26 (reviewer 3 ). All $p$ values exceed 0.05 ; therefore, no significant difference was found between the accuracy values of the single series in ABER position and the conventional MR arthrography.

A scatter plot is drawn which represents the overall accuracy of MR arthrography based on ABER versus conventional MR arthrography confirmed by arthroscopy (Fig. 5).
Table 3250 MR arthrograms: inter-observer agreement

\begin{tabular}{llccl}
\hline & & \% Agreement & $\kappa$ value & Interpretation \\
\hline ABER & Reviewer 1 vs. Reviewer 2 & 75 & 0.556 & Moderate \\
& Reviewer 1 vs Reviewer 3 & 76 & 0.562 & Moderate \\
& Reviewer 2 vs Reviewer 3 & 67 & 0.438 & Moderate \\
Conventional MR & Reviewer 1 vs Reviewer 2 & 78 & 0.624 & Good \\
arthrography & Reviewer 1 vs Reviewer 3 & 76 & 0.575 & Moderate \\
& Reviewer 2 vs Reviewer 3 & 66 & 0.441 & Moderate \\
\hline
\end{tabular}


Table 4 Study group (92 MR arthrograms): results of ABER compared with arthroscopy

\begin{tabular}{|c|c|c|c|c|c|c|}
\hline & \multicolumn{6}{|l|}{ ABER } \\
\hline & Intact & Bankart & Perthes & ALPSA & Lesions NOS & Not assessable \\
\hline \multicolumn{7}{|l|}{ ARTHROSCOPY } \\
\hline Intact (45) & $40 / 37 / 41$ & $3 / 6 / 0$ & $0 / 2 / 1$ & $1 / 0 / 1$ & $0 / 0 / 0$ & $1 / 0 / 2$ \\
\hline Bankart (8) & $0 / 0 / 1$ & $2 / 2 / 1$ & $1 / 4 / 1$ & $4 / 2 / 5$ & $1 / 0 / 0$ & $0 / 0 / 0$ \\
\hline Perthes (7) & $2 / 1 / 2$ & $2 / 1 / 2$ & $3 / 5 / 1$ & $0 / 0 / 2$ & $0 / 0 / 0$ & $0 / 0 / 0$ \\
\hline Alpsa (29) & $5 / 4 / 2$ & $3 / 10 / 5$ & $8 / 13 / 8$ & $11 / 2 / 13$ & $2 / 0 / 0$ & $0 / 0 / 1$ \\
\hline Lesions NOS (3) & $0 / 0 / 0$ & $0 / 2 / 0$ & $0 / 1 / 1$ & $2 / 0 / 2$ & $1 / 0 / 0$ & $0 / 0 / 0$ \\
\hline
\end{tabular}

*Reviewer $1 / 2 / 3$, correct correlations in bold of $96 \%$ and a specificity of $97 \%$ compared with $48 \%$ and 91\% using conventional MR arthrography series. Although accuracy may be increased by performing an additional series in ABER position to conventional MR arthrography, scan time will be prolonged by $15 \mathrm{~min}$, due to repositioning of patient and coil and subsequent scanning.

According to the arthroscopic results of this study, 45 of 92 patients had an intact anterior labrum, and in 44 of 92 patients, an anterior labroligamentous lesion was diagnosed. Eight of 44 labroligamentous lesions (18\%) were classified by arthroscopy as a Bankart lesion, seven (16\%) as a Perthes lesion and $29(66 \%)$ as an ALPSA lesion. In three patients, an unclassifiable labral lesion was detected. In comparison to other studies [20-22], we found a remarkable high number of ALPSA lesions, which can be explained by the large number of patients with chronic recurrent dislocations among our patient population.

Based on the single series in ABER position, the correct classification i.e. normal labrum, Bankart, Perthes and ALPSA lesion was established in $50-62 \%$ of cases, versus percentages of $53-63 \%$ based on the conventional MR arthrography. No significant differences were found between both protocols. In the present study, a maximum of two out of seven $(29 \%)$ Perthes lesions were diagnosed on the conventional MR arthrography and a maximum of five out of seven (71\%) Perthes lesions were diagnosed on the single series in ABER position. This is in accord with the hypothesis stated by Wischer et al. [15] that ABER positioning may increase the detection of Perthes lesions. A Perthes lesion represents a labral tear without disruption
Table 5 Study group (92 MR arthrograms): results of conventional MR arthrography compared with arthroscopy

*Reviewer $1 / 2 / 3$, correct correlations in bold

\begin{tabular}{|c|c|c|c|c|c|c|}
\hline & \multicolumn{6}{|c|}{ Conventional MR arthrography } \\
\hline & Intact & Bankart & Perthes & ALPSA & Lesions NOS & Not assessable \\
\hline \multicolumn{7}{|l|}{ Arthroscopy } \\
\hline Intact (45) & $40 / 38 / 38$ & $1 / 4 / 1$ & $0 / 0 / 1$ & $3 / 3 / 5$ & $0 / 0 / 0$ & $1 / 0 / 0$ \\
\hline Bankart (8) & $0 / 0 / 0$ & $3 / 4 / 1$ & $0 / 0 / 0$ & $4 / 4 / 7$ & $1 / 0 / 0$ & $0 / 0 / 0$ \\
\hline Perthes (7) & $1 / 0 / 0$ & $4 / 4 / 5$ & $2 / 1 / 0$ & $0 / 2 / 2$ & $0 / 0 / 0$ & $0 / 0 / 0$ \\
\hline Alpsa (29) & $4 / 2 / 2$ & $11 / 20 / 3$ & $1 / 1 / 1$ & $13 / 6 / 22$ & $0 / 0 / 0$ & $0 / 0 / 1$ \\
\hline Lesions NOS (3) & $0 / 0 / 1$ & $1 / 2 / 0$ & $0 / 0 / 0$ & $2 / 1 / 1$ & $\mathbf{0} / \mathbf{0} / \mathbf{1}$ & $0 / 0 / 0$ \\
\hline
\end{tabular}


Table 6 Study group (92 MR arthrograms): sensitivity and specificity in percentages

\begin{tabular}{|c|c|c|c|c|c|c|}
\hline & \multicolumn{2}{|c|}{ Sensitivity (\%) } & \multirow[t]{2}{*}{$P$ values } & \multicolumn{2}{|c|}{ Specificity (\%) } & \multirow[t]{2}{*}{$P$ values } \\
\hline & ABER & Conventional MR arthrography & & ABER & Conventional MR arthrography & \\
\hline Reviewer 1 & 85.1 & 89.4 & 0.69 & 88.9 & 88.9 & 1.00 \\
\hline Reviewer 2 & 89.4 & 95.7 & 0.38 & 82.2 & 84.4 & 1.00 \\
\hline Reviewer 3 & 87.2 & 91.5 & 0.75 & 91.1 & 84.4 & 0.45 \\
\hline
\end{tabular}

${ }^{*} p<0.05$ : significant

of the capsule. In neutral position this may result in a (near) normal appearance of the anterior labrum. In ABER position due to provocation a pouch-like distension of the capsule is more accentuated.

Unfortunately, a correct pre-operative distinction between a displaced (ALPSA) and a nondisplaced labrum (Bankart, Perthes) could not always be accomplished using ABER or conventional MR arthrography; therefore, a correct preoperative selection of the type of operative intervention was not always achievable. However, this study does demonstrate that a single series in ABER position is as accurate as conventional MR arthrography in distinguishing an intact anteroinferior labrum from an anteroinferior labroligamentous lesion; therefore, it can be concluded that a single series in ABER position can accurately predict whether an operative intervention will be necessary or not.

Provided that the orthopaedic surgeon is interested in a quick and reliable survey of the shoulder for the early detection of soft tissue damage, like anteroinferior labroligamentous lesions, in patients suffering from acute anterior glenohumeral instability, a single MR arthrography series in ABER position as diagnostic tool can be considered. This reduced scan protocol will save scan time, and, besides the detection of labral-ligamentous lesions, it is reliable in diagnosing (partial thickness) rotator cuff lesions and Hill Sachs lesions as well [17, 23, 24].

In exploring bony changes of the glenoid, a single series in ABER position is assumed to be of limited value. To detect and determine (semi)quantification of glenoid defects, which may have consequences for surgical strategy, a series in the oblique sagittal plane should be added to the protocol [25].

ABER is assumed to be of limited value in detecting injuries of the posterior labrum as well, including reverse Bankart lesions and posterior labrocapsular sleeve avulsions (POLPSAs); therefore, a single series in ABER position may not be an appropriate diagnostic tool in patients with posterior instability.

It should be noticed, however, that some patients may not tolerate ABER positioning due to instability or pain. According to Cvitanic et al. [14], 20\% of patients suffering from shoulder pain or instability are unable to undergo ABER positioning. In our experience, the number of patients that could not be evaluated in ABER position does not exceed $8 \%$ of the total group of shoulder MR arthrograms.

Another disadvantage is that the arm positioned in abduction external rotation may be more susceptible to motion artefacts than the arm positioned in neutral position. Also, positioning of the surface coil can be more difficult resulting

Fig. 5 Overall accuracy

Overall accuracy

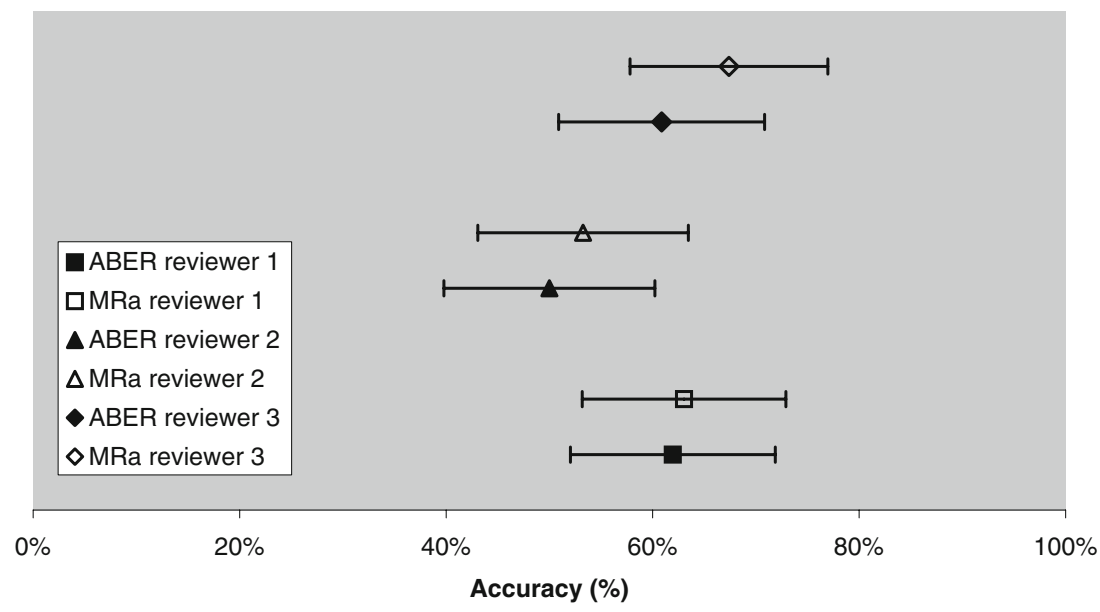


in an off-centre position of the area of interest. An adequate amount of contrast medium is relevant, particularly for optimal view of the articular margins of rotator cuff.

A limitation of this study is that anteroinferior labroligamentous lesions were subclassified in Bankart, Perthes and ALPSA lesions exclusively. Bankart, ALPSA and Perthes lesions are by far the most frequently encountered lesions in patients with shoulder instability. Therefore, incidental/uncommonly appreciated labroligamentous lesions, like a HAGL and a GLAD, were not registered specifically. Presence or absence of the middle glenohumeral ligament, playing a minor role in the stability of the shoulder joint, was also not registered. The final limitation to be mentioned is the difference in both slice thickness and angle being used to perform the true axial T1-weigthed sequence in neutral position (4 $\mathrm{mm}$ slice thickness) and the oblique axial $\mathrm{T} 1$-weigthed fat-suppressed sequence in ABER position ( $3 \mathrm{~mm}$ slice thickness), both in favour of the performance of the single series in ABER position.

We conclude that a single fat-suppressed T1-weighted MR arthrography series in ABER position is as accurate as conventional MR arthrography in detecting anteroinferior labroligamentous lesions.

Open Access This article is distributed under the terms of the Creative Commons Attribution Noncommercial License which permits any noncommercial use, distribution, and reproduction in any medium, provided the original author(s) and source are credited.

\section{References}

1. Soslowsky LJ, Flatow EL, Bigliani LU, Mow VC. Articular geometry of the glenohumeral joint. Clin Orthop Relat Res. 1992; 285: 181-190.

2. Pollock RG, Flatow EL. Classification and evaluation. In: Biglian LU, editor. The unstable shoulder. Rosemount, Ill: American Academy of Orthopaedic Surgeons; 1996. p. 25-36.

3. Chandnani VP, Yeager TD, DeBerardino T, et al. Glenoid labral tears prospective evaluation with MRI imaging, MR arthrography, and CT arthrography. Am J Roentgenol. 1993; 16: 1229-1235.

4. Chandnani VP, Gagliardi JA, Murnane TG, et al. Glenohumeral ligaments and shoulder capsular mechanism: evaluation with MR arthrography. Radiology. 1995; 196: 27-32.

5. Palmer WE, Brown JH, Rosenthal DI. Labral-ligamentous complex of the shoulder: evaluation with MR arthrography. Radiology. 1994; 190: 645-651.

6. Flannigan B, Kursunoglu-Brahme S, Snyder S, Karzel R, Del Pizzo W, Resnick D. MR arthrography of the shoulder comparison with conventional MR imaging. AJR Am J Roentgenol. 1990; 155: 829-832.

7. Tirman PF, Palmer WE, Feller JF. MR arthrography of the shoulder. Magn Reson Imaging Clin N Am. 1997; 5: 811-839.

8. Waldt S, Burkart A, Imhoff AB, Bruegel M, Rummeny EJ, Woertler K. Anterior shoulder instability: accuracy of MR arthrography in the classification of anteroinferior labroligamentous injuries. Radiology. 2005; 237: 578-583.
9. Legan JM, Burkhard TK, Goff WB 2nd. Tears of the glenoid labrum: MR imaging of 88 arthroscopically confirmed cases. Radiology. 1991; 179: 241-246.

10. Beltran J, Rosenberg ZS, Chandnani VP, Cuomo F, Beltran S, Rokito A. Glenohumeral instability: evaluation with MR arthrography. RadioGraphics. 1997; 17: 657-673.

11. Song H-T, Huh Y-M, Kim S. Anterior-inferior labral lesions of recurrent shoulder dislocation evaluated by MR arthrography in an adduction internal rotation (ADIR) position. Journal of Magn Reson Im. 2005; 23(1): 29-35.

12. Wintzell G, Larsson H, Larsson S. Indirect MR arthrography of anterior shoulder instability in the ABER and the apprehension test positions a prospective comparative study of two different shoulder positions during MRI using intravenous gadodiamide contrast for enhancement of the joint fluid. Skeletal Radiol. 1998; 27: 488-494.

13. Kwak SM, Brown RR, Trudell D, Resnick D. Glenohumeral joint: comparison of shoulder positions at MR arthrography. Radiology. 1998; 208: 375-380.

14. Cvitanic O, Tirman PF, Feller JF, Bost FW, Minter J, Carroll KW. Using abduction and external rotation of the shoulder to increase the sensitivity of MR arthrography in revealing tears of the anterior glenoid labrum. AJR Am J Roentgenol. 1997; 169: 837-844.

15. Wischer TK, Bredella MA, Genant HK, Stoller DW, Bost FW, Tirman PFJ. Perthes lesion (a variant of the Bankart lesion): MR imaging and MR arthrographic findings with surgical correlation. AJR Am J Roentgenol. 2002; 178(1): 233-237.

16. Choi JA, Suh SI, Kim BH. Comparison between conventional MR arthrography and abduction and external rotation MR arthrography in revealing tears of the antero-inferior glenoid labrum. Korean J Radiol. 2001; 2: 216-221.

17. Tirman PF, Bost FW, Steinbach LS. MR arthrographic depiction of tears of the rotator cuff benefit of abduction and external rotation of the arm. Radiology. 1994; 192: 851-856.

18. Bankart ASB. Recurrent or habitual dislocation of the shoulder joint. Br Med J. 1923; 2: 1132-1133.

19. Perthes G. Uber Operationen bei habitueller schulterluxation. Deutsch Ztschr Chir. 1906; 85: 199-227.

20. Neviaser TJ. The anterior labroligamentous periosteal sleeve avulsion lesion: a cause of anterior instability of the shoulder. Arthroscopy. 1993; 9: 17-21.

21. Antonio GE, Griffith JF, Yu AB, Yung PS, Chan KM, Ahuja AT. First-time shoulder dislocation. High prevalence of labral injury and age-related differences revealed by MR arthrography. J Magn Reson Imaging. 2007; 26(4): 983-991.

22. Yiannakopoulos CK, Mataragas E, Antonogiannakis E. A comparison of the spectrum of intra-articular lesions in acute and chronic anterior shoulder instability. Arthroscopy. 2007; 23(9): 985-990.

23. Lee SY, Lee JK. Horizontal component of partial-thickness tears of rotator cuff: imaging characteristics and comparison of ABER view with oblique coronal view at MR arthrography-initial results. Radiology. 2002; 224: 470-476.

24. Schreinemachers SA, Hulst van der VPM, Willems WJ, Bipat S, Woude van der HJ. Detection of partial-thickness supraspinatus tendon tears: is a single direct MR arthrography series in ABER position as accurate as conventional MR arthrography? Skeletal Radiol 2009; Mar 18: Epub ahead of print.

25. Huijsmans PE, Haen PS, Kidd M, Dhert WJ, van der Hulst VP, Willems WJ. Quantification of a glenoid defect with threedimensional computed tomography and magnetic resonance imaging: a cadaveric study. Shoulder Elbow Surg. 2007; 16(6): 803-809. 